\title{
Bibliotheken in der Mitte unserer Gesellschaft: Teilhabe an Bildung, Gesellschaft und Kultur
}

\author{
Prof. Andreas Degkwitz zum neuen Bundesvorsitzenden des \\ Deutschen Bibliotheksverbandes gewählt
}

http://doi.org/10.1515/bd-2019-0046

Die Ergebnisse der diesjährigen Vorstandwahlen wurden auf der Mitgliederversammlung des Deutschen Bibliotheksverbands e.V. (dbv) am 20.03.2019 in Leipzig bekanntgegeben. Mit großer Mehrheit ist Prof. Andreas Degkwitz, Direktor der Universitätsbibliothek der Humboldt-Universität zu Berlin, mit 805 von 878 gültigen Stimmen zum neuen Vorsitzenden gewählt worden. Wahlberechtigt bei der Briefwahl waren Vertreter von mehr als 2.000 Mitgliedsbibliotheken, die Wahlbeteiligung lag bei $50 \%$. Degkwitz tritt die Nachfolge von Barbara Lison, Direktorin der Stadtbibliothek Bremen, an, die den Verband von 2016 bis 2019 geführt hat und aktuell zur IFLA Präsidentin gewählt wurde.

Der Präsident des Deutschen Bibliotheksverbandes, Dr. Frank Mentrup, dankte auf der Mitgliederversammlung dem bisherigen Vorstand und begrüßte die neuen Vorstandsmitglieder: „Ich freue mich auf eine lebendige Zusammenarbeit mit Prof. Degkwitz und den gewählten Vorstandsmitgliedern des Verbands und will mich für eine weiterhin starke Interessenvertretung für alle Bibliotheken in Deutschland einsetzen.“

Der neue Verbandsvorsitzende Andreas Degkwitz ergänzte auf der Mitgliederversammlung: „Verstärkt übernehmen Bibliotheken Aufgaben der Teilhabe an Bildung, Gesellschaft und Kultur. Hinzu kommt Kompetenzvermittlung zur digitalen Aneignung, Produktion und Weitergabe von Wissen. Öffentliche und wissenschaftliche Bibliotheken greifen diese Herausforderungen aktiv auf. Der digitale Wandel positioniert Bibliotheken in der Mitte unserer Gesellschaft.“

In den dbv-Bundesvorstand 2019-2022 wurden außerdem Elisabeth Sträter, Direktorin der Stadtbibliothek im Bildungscampus Nürnberg, Petra Büning, Leiterin der Fachstelle für Öffentliche Bibliotheken NRW und Marion Mattekat, Direktorin der Stadt- und Landesbibliothek Potsdam (SLB) für den Bereich Öffentliche Bibliotheken und Petra Hätscher, Direktorin des Kommunikations-, Informations-, Medienzentrums der Universität Konstanz (KIM), Frank Scholze, Direktor der Bibliothek des Karlsruher Instituts für Technologie (KIT) und Dr. Jochen 
Johannsen, Direktor der Universitätsbibliothek Siegen für den Bereich wissenschaftliche Bibliotheken, gewählt.

\section{Kontakt:}

Deutscher Bibliotheksverband e.V. (dbv)

Maiken Hagemeister, Pressesprecherin und Leitung Kommunikation, Tel.: 0 30/644 9899 25, E-Mail: hagemeister@bibliotheksverband.de, http://www.bibliotheksverband.de, http://www.bibliotheksportal.de 